segments were used to classify segments as low or high molecular disease activity (MDA). Agreement between endoscopy/histology and MDA was evaluated by Cohen's kappa coefficient ( $\square$ ) and $\%$ agreement.

Results In segments with BL GHAS activity subscore $\geq 2$, Ep (10-30\%) and non-Ep (25-35\%) MDA were elevated and correlated to histologic activity. Table 1 shows the effect of FIL on MDA in the intestinal mucosa: significantly fewer low BL MDA segments showed MDA worsening, and significantly more high BL MDA segments showed MDA improvement (pSTAT3 only) with FIL vs. PBO. Agreement for MDA and endoscopy was fair to moderate ( $\kappa 0.3-0.5)$, and for MDA and histology was moderate to good ( $\kappa 0.4-$ $0.8)$.

Conclusions FIL improved JAK1-related MDA within the mucosa of pts with CD. Agreement between MDA and clinical indices was highest with histology.

\section{P118 POSITIVITY THRESHOLDS OF TOTAL INFLIXIMAB AND ADALIMUMAB ANTI-DRUG ANTIBODY ASSAYS AND IMPACT IN CLINICAL PRACTICE}

${ }^{1}$ Rachel Nice*, 2,3 ${ }^{2}$ eil Chanchlani, ${ }^{2,3}$ Nicholas A Kennedy, ${ }^{3}$ Harry D Green, ${ }^{2,3}$ Simeng Lin ${ }^{2,3}$ Claire Gordon, ${ }^{2,3}$ Desmond Chee, ${ }^{2,3}$ Benjamin Hamilton, ${ }^{3}$ Claire Bewshea, 2,3 James Goodhand, ${ }^{2,3}$ Tariq Ahmad, 'Timothy McDonald, 'Mandy Perry. ${ }^{1}$ Exeter Laboratory, RDandE NHS Foundation Trust, Exeter, UK; ${ }^{2}$ Department of Gastroenterology, RDandE NHS Foundation Trust, Exeter, UK; ${ }^{3}$ Exeter IBD Research Group, University of Exeter, Exeter, UK

\subsection{6/gutjnl-2020-bsgcampus. 193}

Introduction Anti-drug antibodies can affect biopharmaceutical pharmacokinetics by increasing or decreasing drug clearance. Drug-tolerant (total), unlike drug-sensitive (free), antibody assays permit antibodies to be measured in the presence of drug.

We aimed to confirm the positivity threshold of our total anti-tumour necrosis factor (TNF) antibody ELISA assays in healthy volunteers and to use this threshold to report the prevalence of clearing and transient antibodies in patients treated with infliximab and adalimumab.

Methods Serum was obtained from 498 anti-TNF-naïve healthy adults recruited to the Exeter 10,000 study and tested for total anti-drug antibodies to infliximab and adalimumab. We used bootstrapping to calculate the $80 \%$ one-sided lower confidence interval $[\mathrm{CI}]$ of the 99th centile recommended by the FDA to define assay thresholds.

We used paired drug and anti-drug antibody levels derived from our national therapeutic drug monitoring service to report the distribution of clearing (antibody positive, drug negative) vs non-clearing (antibody positive, drug positive) antibodies. In patients with at least two test results, antibodies were classified as transient (single positive test with subsequent negative test) or persistent (at least two positive tests).

Results The $80 \%$ one-sided lower CI of the 99th centile titre for total anti-drug antibody to infliximab and adalimumab were $8.7 \mathrm{AU} / \mathrm{mL}$ and $5.9 \mathrm{AU} / \mathrm{mL}$, respectively.

Using these thresholds, at the time of last testing, of 7,428 and 4,043 patients treated with infliximab and adalimumab; $21.1 \%$ and $8.3 \%$ had clearing antibodies and $27.9 \%$ and $20.0 \%$ had non-clearing antibodies, to infliximab and adalimumab, respectively.

Amongst patients with at least two tests, most developed persistent antibodies. Irrespective of anti-TNF drug, or

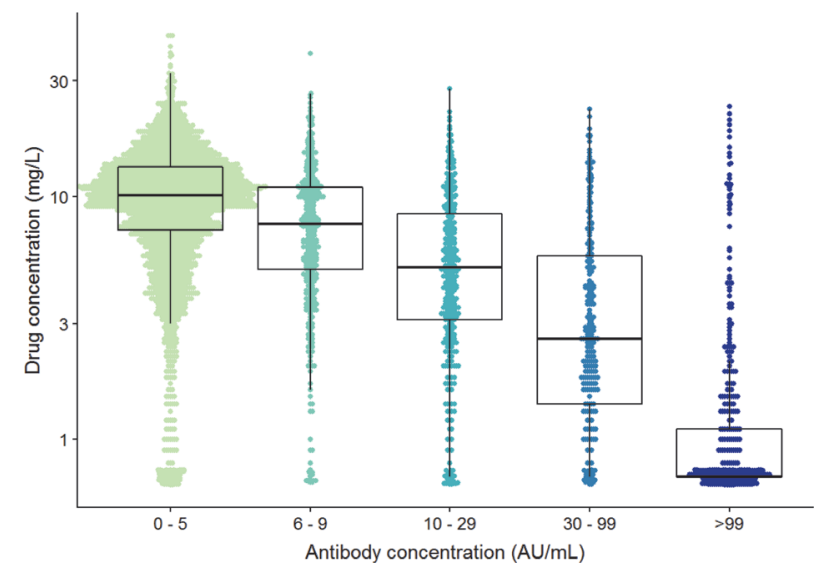

Abstract P118 Figure 1 Relationship between adalimumab drug and anti-drug antibody levels in national TDM cohort

threshold used, less than $10 \%$ patients developed transient antibodies.

Across both our national TDM cohort and the PANTS study, there were significant associations between anti-drug antibody and drug levels (figure 1). In PANTS, higher antidrug antibody levels were associated with poorer outcomes at weeks 14 and 54 .

Conclusions We report lower positivity thresholds for the IDKmonitor ${ }^{\circledR}$ total anti-TNF antibody ELISA assays than the manufacturer, in particular, for adalimumab. Transient antibody formation is uncommon: most patients develop persistent antidrug antibodies that lead to drug clearance.

\section{P119 RISK OF FURTHER SURGERY AND ADHERENCE TO COLONOSCOPY GUIDELINES FOLLOWING RIGHT HEMI- COLECTOMY FOR CROHN'S DISEASE}

${ }^{1}$ Dominic King*, ${ }^{2}$ Benjamin Coupland, ${ }^{2}$ Aman Dosanjh, ${ }^{2}$ Prashant Patel, ${ }^{1}$ Nigel Trudgill. ${ }^{1}$ Sandwell and West Birmingham Hospitals NHS Trust, UK; ${ }^{2}$ Informatics Dept University Hospitals Birmingham, UK

\subsection{6/gutjnl-2020-bsgcampus. 194}

Introduction The risk of further surgery following right hemicolectomy (RH) for Crohn's disease (CD) is high ( 40\%). Recent guidelines advise colonoscopy 6-12 months following $\mathrm{RH}$ to reduce the risk of further surgical intervention through medical therapy. We examined the risk of further surgery and use of colonoscopy following index RH.

Methods Hospital Episode Statistics were used to identify subjects with CD and RH between 2007 and 2016 in England. Adherence to post resection colonoscopic assessment guidance and risk of further surgery at the same site were investigated. Cox regression models examined the risk factors associated with further surgery and funnel plots demonstrated the colonoscopy practice of providers.

Results 12,230 CD subjects (55\% female, median age 36 (IQR 26-49) years) had a RH during the study period. 1,367 (11\%) had further surgery at the anastomotic site during follow up. $40 \%$ of Index surgery and $50 \%$ of further surgery was performed during an elective admission. 9\% $(747 / 8,293)$ of those with 5 year at follow up had further surgery as and $17 \%(366 / 2,163)$ of those with 10 years at follow up. Age over 54 compared to 18-24 years had a reduced risk of further surgery (adjusted Hazard ratio 0.81 (95\%CI 0.67-0.97), 